\title{
EFFECTIVE OUTAGE PLANNING AND IMPLEMENTATION FOR NUCLEAR POWER PLANTS IN THE UK
}

\author{
Holley Reece-Barkell ${ }^{1}$ and Willem J J Vorster ${ }^{2}$ \\ ${ }^{1}$ Frazer-Nash Consultancy Limited, UK \\ ${ }^{2}$ EDF Energy Nuclear Generation Ltd, UK
}

\begin{abstract}
Effective outage planning and implementation is critical to the efficient and safe operation of commercial nuclear power plants in the UK. Outages are necessary for refuelling, for preventive and corrective maintenance and for major modification and improvement projects. Outages involve the support of many organisations working together and hence require high levels of coordination. Planning of activities before an outage is critical to their overall success.

Establishing the integrity of power plant piping and pressure vessel components is a key objective of any outage and is the subject of this paper. It requires an understanding of the specific threats, their relationship to the overall plant conditions and the mitigating measures required to assure safe operation. Understanding the specific threats allows the engineering function of an organisation to advise on the component Minimum Acceptable Thicknesses (MATs) which can be used to assure integrity via comparison with measurements taken during outage inspections. Historically a variety of methodologies have been used to provide MATs including design drawing specified thicknesses and design code based or fitness-for-purpose methods. It is important that a robust procedure be applied to promote consistency of approach across all power station assets.
\end{abstract}

This paper provides an overview of the strategy and methodologies employed to determine MATs which ensure that, over a specified inspection interval, a component would not fail by plastic collapse, creep rupture, fatigue, ratcheting or buckling and if the weld were to contain a crack-like defect at the time of inspection, it would not grow to a limiting size within the inspection interval.

Readers of this paper will gain a valuable insight into the statutory outage process applicable to nuclear power plants in the UK. A particular focus of this paper is on the structural integrity assessments applied in a non-traditional sense prior to, during and after the statutory outage. As well as sharing a valuable insight into the assessment methodologies this paper highlights best industrial practice.

\section{BACKGROUND}

Statutory outages are essential for the continued safe operation of nuclear power plants. During outages the reactors are offline and hence detailed planning and monitoring is required to return the reactors to power when planned to minimise commercial losses without compromising nuclear safety. Each Advanced Gas Cooled Reactors (AGRs) typically has an outage period every three years, with preparation activities commencing approximately two years before. The duration of the outage itself typically lasts two to three months, but this varies depending on the nature of the inspection, maintenance, repair and replacement work required. Work contributing to the outage is conducted according to company arrangements agreed by the UK safety regulator, the Office for Nuclear Regulation (ONR). 
This paper focuses on the structural integrity assessments undertaken as part of the outage planning process, which includes the calculation of Minimum Acceptable Thicknesses (MATs). Since 1998 a consistent assessment methodology and process has been applied to UK AGRs to determine MATs. These MATs ensure that, over a specified inspection interval, were the weld/component to be defect free, it would not fail due to any of the relevant failure mechanisms, which are typically plastic collapse, creep rupture, fatigue, incremental collapse (ratcheting) or buckling. Furthermore, if the weld/component were to contain a crack-like defect at the time of inspection, A MAT could be calculated to demonstrate that the crack will not grow to a limiting size within the inspection interval via a defect tolerance and fatigue crack growth calculation. This consistent approach to MAT assessments brings substantial benefits including a reduction in the number of 'unnecessary' structural integrity related referrals during the outage period and a greatly reduced risk that referral clearance might delay return to service of the reactor. The approach taken to consistently calculate MAT values for defect free components is described in this paper, together with supporting outage preparation activities and a case study.

\section{PRE STATUTORY OUTAGE PREPARATION}

Before MATs can be determined, the components for which assessments are required must be identified. This is achieved via the Intentions Proposal Document (IPD). The IPD lists the components which are due to be inspected as part of a non-destructive testing In-Service Inspections (ISI) programme during the statutory outage and identifies the appropriate inspection procedure (UT, MPI for example) which will be utilised in each case. The IPD then identifies which of the components due to be inspected require the calculation of MATs prior to the inspection. Components are selected for inspection for a variety of reasons driven by the requirement to demonstrate nuclear and industrial safety of the plant.

As part of the process for recommending components for inspection, a Creep Life Review (CLR) is undertaken, which identifies components on the steam pipework systems with a high predicted creep rupture damage at the next or next but one statutory outage. Such components are recommended to the Design Authority for inspection and as a result may be included in the inspection programme described in the IPD. The creep rupture damage calculations are based on Mean Effective Creep Temperatures (MECTs) and Mean Steam Pressures (MSPs). The MECT is a single temperature value which, at the appropriate MSP, produces the same creep rupture damage as the summation of all of the creep rupture damages over the operational life of the plant item. The CLR, MECT and MSP reports are produced typically on a yearly basis as part of the ongoing structural integrity assessments.

Once the components requiring inspection have been selected, it is necessary to identify which ones require MATs. Components requiring MATs are defined by a number of factors such as accessibility, type of inspection and whether thicknesses which can be compared to the MAT are due to be measured. Prior to commencement of the statutory outage, the MATs are uploaded into the Outage Management Database (OMD) ready for use during the outage period. There are a number of other outage preparation tasks, such as hanger surveys and non-ISI activities for example. However, these are not the subject of this paper and hence are not discussed further here.

\section{COMMITMENTS DURING THE STATUTORY OUTAGE}

During the statutory outage itself, the results of the component inspections are typically assessed by Inspection and Materials specialists. Acceptance of the inspection results is typically made in accordance with Assessment Guidesheets which define criteria such as restrictions to test and the acceptability of defect indications, noting a certain level of operating and inspection experience is also required. The Guidesheets also define criteria for the acceptance of measured thicknesses. If after comparing measured thicknesses to the MAT the thickness is deemed unacceptable, the component should be referred, deemed a 'referral', to Structural Integrity specialists for further more detailed assessment. 
Components may also be referred for further assessment if a MAT was not provided prior to the outage (for example if an additional inspection was added to the programme), if a component contained a defect indication which is not acceptable to the Guidesheets or if the surface profile of a component is reported to be unusual. Structural Integrity specialists assess the referred component and either provide a return to service argument, or may if needed, recommend a repair or replacement strategy.

\section{POST STATUTORY OUTAGE COMMITMENTS}

Prior to reactor return to service, the inspection results are typically formally agreed via an Engineering Change (EC) document which is submitted to the ONR for agreement. There may be other requirements, such as a full outage report, which follow this, however, by this time, the structural integrity assessments will be complete, or recommendations and commitments for future work will have been made.

\section{MAT CALCULATION METHODOLOGY}

A stated above, two groups of components requiring MATs can be described as those having a frequent or infrequent nuclear safety duty class. For frequent safety class components, consideration must be given to faults with a frequency of more than $10^{-3}$ per reactor year, for infrequent items, this is between $10^{-3}$ and $10^{-5}$ per reactor year. Frequent and infrequent components can be further divided into those that are potentially susceptible to FAC and those that are non-FAC. The FAC and non-FAC components are typically subject to deadweight, pressure and cyclic loading (thermal and pressure) and hence may fail due to plastic collapse, ii) ratcheting, iii) fatigue or iv) buckling. For non-FAC components, failure by creep rupture may also require consideration, depending on the operating temperature. However, it is not considered further here. FAC components do not operate at temperatures for which creep rupture is a concern.

A minimum required thickness is determined for each relevant failure mechanism. The minimum required thicknesses are calculated using a combination of design code requirements and Fitness for Purpose (FFP) assessments, which are described in the following sections. The methodology varies depending on the component types, such as a straight pipe or branch for example.

In most cases, the design code and Fitness for Purpose methodologies are designed to produce a stress or stress range that can be compared to an allowable stress or stress range. To calculate minimum required thicknesses, it is often necessary to rearrange the equations or iterate to a solution, whereby the calculated stress or stress range is equated to the allowable.

\section{DESIGN CODE CALCULATIONS}

Some of the pipework systems within UK AGR plant were principally designed to BS 806: 1967 . The code has since been updated, with the most recent version being released in 1993 [3]. BS 806 was withdrawn in 2002 and partially superseded by the harmonised code BS EN 13480 [1]. In particular, part three of the code, BS EN 13480-3, is used to determine the majority of required thicknesses.

\section{Pressure Scantling Calculations}

For scantling calculations the design stress can be determined in accordance with Section 5.2.1.1 of BS EN 13480 [1]. Section 4.5 of the code requires that a joint coefficient is included for pipework that contains butt welds other than circumferential ones. For seam welded pipes the joint coefficient should be set to between 0.7 and 1.0 depending on what non-destructive testing (NDT) has been conducted. As information regarding the NDT conducted during construction is not always readily available, a joint 
coefficient of 0.7 can conservatively be assumed in calculations, except for components constructed from seamless pipes. In these cases the joint coefficient can be omitted (i.e. given a value equal to unity).

Straight pipe sections and butt welds can be assessed in accordance with Section 6.1 of BS EN 13480-3 [1]. Elbows and smooth pipe bends can be assessed in accordance with Section 6.2.3.1 of BS EN 134803 [1], with required thicknesses at the extrados and intrados determined respectively. All bends should account for the bend radii appropriately. Where drawings do not specify a bend radius, a potentially conservative short radius bend in accordance with BS 1640-3:1968 [4] should be considered. Mitre bends should be assessed in accordance with Section 6.3 of BS EN 13480-3 [1] in which the maximum mitre angle $(\theta)$ allowed by the code, $22.5^{\circ}$, can conservatively be used.

Branches can be assessed using the pressure area method of BS EN 13480-3 [1]. Generally it can be assumed that the ratio between the branch and main pipe thicknesses remains constant as the component thickness is reduced. Branches that are in close enough proximity that they could potentially interact should be checked against the isolation requirements of Section 8.4.1 of BS EN 13480-3 [1]. Where interaction is a possibility assessment using Section 8.5.2 of BS EN 13480-3 [1] can be undertaken. Branches on bends can be assessed in accordance with BS EN 13480-3 via scaling methods.

Some pipework branches cannot be classified as typical, for example Y-Pieces. In such cases the pressure area method described in Equation 8.4.3-3 of BS EN 13480-3 [1] can be used to calculate an internal pressure scantling thickness. The pipework can be approximated as a cylindrical Y-piece as described in Section 8.6.1 of BS EN 13480-3 [1], with areas calculated in accordance with Figure 8.6.1-1 of the code. Section 8.6.3 of BS EN 13480-3 provides a method for assessing triform reinforced branches, but it requires the dimensions and material specification of the reinforcement.

Reducers can be assessed using the methodology in Section 6.4 of BS EN 13480-3 [1]. At the larger end of the reducer, minimum required thicknesses can be calculated for the straight pipe using Equations 6.11 and 6.1-2, the conical shell using equations 6.4.4-1 and 6.4.4-2, and the junction between the large end of the cone and the cylinder using equations 6.4.6-1 and 6.4.6-2, and the larger of these three values retained. At the smaller end of the reducer, minimum required thicknesses can also be calculated for the straight pipe and the conical shell, along with the junction between the small end of the cone and the cylinder using the methodology in Section 6.4.8.2, and the largest of these three values retained.

\section{Flexibility Calculations}

Minimum required thicknesses to meet the flexibility requirements of Section 12 of BS EN 13480-3 [1] can be calculated for both sustained (deadweight) and internal pressure loading and for stress ranges due to thermal expansion or alternating loads. Stress intensification factors should be applied, for each component type. Equation 12.3.2-1 of BS EN 13480-3 can be been used to calculate the required thickness to avoid failure due to sustained loading, taking account of axial pressure loads and sustained mechanical loads (i.e. self weight). The design stress for flexibility analysis can be determined as described in Section 12.3.3 of the code. Applied system moments should be extracted from the sustained (non-intensified deadweight) load cases of appropriate pipe stress models. Required thicknesses to avoid failure due to thermal expansion can be calculated using equation 12.3.4-2 of BS EN 13480-3 [1], which limits the total axial stress due to sustained load and thermal expansion to the sum of the design stress at operating temperature and the allowable stress range. The allowable stress range can be calculated using Equation 12.1.3-1 of the code. The stress range reduction factor can conservatively be set to 1 , and this means that meeting the design code requirements to avoid ratcheting (as well as demonstrating compliance with other flexibility and scantling requirements) implies a fatigue life of 7,000 full pressure and temperature cycles. System moment ranges should be extracted from the relevant thermal stress range load cases of appropriate pipe stress models. The system moments extracted from such models are 
likely to have been calculated to BS 806 [3] rather than BS EN 13480 [1]. The flexibility factors are not identical between the two codes, and as such there will be some differences in the moments used. These differences are expected to be small and should not have a significant effect on the assessments.

\section{External Pressure/Buckling}

Components may be subjected to external pressure loading. For the purposes of the buckling calculations, where vacuum loading is anticipated but not quantified, a full vacuum of $-0.1 \mathrm{MPa}(\mathrm{g}) \mathrm{can}$ be assumed. Required thicknesses to avoid buckling due to external loading can be calculated using the methodology in Section 9.3.2 of BS EN 13480-3 [1], which requires the unstiffened length of pipe. This dimension should be identified from design drawings as the distance between stiffening features such as valves and flanges. Where insufficient information is available to do this the unstiffened length can be assigned a conservative length, e.g. $100 \mathrm{~m}$. The use of $100 \mathrm{~m}$ effectively provides an upper bound thickness as further increases in the unstiffened length have an insignificant effect on the required thickness. There is no explicit method for calculation of minimum required thicknesses for reducers and branches subject to external pressure within BS EN 13480-3 [1]. In these cases the branch and main, or large and small side of a reducer, can be treated as two separate pipes. Scoping calculations are recommended using clause 8.6.3 of BS EN 13445-3 [5]. This considers vacuum loading of conical shells, and should be used to demonstrate that the minimum required thicknesses calculated assuming separate plain pipes are conservative. BS EN 13480-3 [1] also requires that the thickness of a component under external pressure shall be not less than the thickness required by the standard for similar components under the same internal pressure (and with a joint coefficient equal to 1 even if axial seam welds are present).

\section{API Fitness for Service}

Should the minimum required thickness, calculated using the design code methodology described in the previous section, be small (less than 2.5mm), API 579-1/ASME FFS-1 [6] states that for pipework to be fit for service, its minimum measured thickness should be the greater of $2.5 \mathrm{~mm}$ or 0.2 times its nominal thickness. This value is not related to the structural integrity of a component, but provides a lower thickness limit to minimise the risk of handling damage and prevent continued operation with excessive degradation. It is noted that this limit is not of use for components that are initially very thin by design. The API 579-1 requirements should be considered when determining the MAT.

\section{FITNESS FOR PURPOSE}

As well as providing minimum required thicknesses determined from the design code, minimum required thicknesses should also be calculated using the FFP methodology. The FFP assessment addresses failure by plastic collapse, ratcheting and fatigue and uses a combination of design code calculations and classical methods to derive stresses or stress ranges to evaluate against allowable stresses or stress ranges.

\section{Plastic collapse}

The reference stress limit for calculating minimum required thicknesses to avoid failure by plastic collapse can be taken as two-thirds of the lower bound flow stress, which is the average of the lower bound yield stress and the lower bound ultimate tensile strength at the design or fault temperature.

A suitable reference stress for plain pipes is the combined circular interaction reference stress. This method accounts for both internal pressure and system moment loading. 
The reference stress for branches can be calculated using the Inverse Code Method. Where main and branch materials are different, the allowable stress for the main pipe material should be used. To account for the effect of system moment loading on the reference stress a system load factor should be calculated for the branch and for the main pipe and the lowest value used. In cases where the branch system moment is unknown, a value of 0.9 can conservatively be applied. In cases where there is zero or insignificant branch moment loading, a factor of 1 should be applied for the branch calculations. Neighbouring branches should be checked to identify those that are susceptible to interaction.

The minimum required thicknesses to resist plastic collapse for smooth bends may be calculated for both thin and thick walled bends. Solutions are typically available for both pressure only loading and combined pressure and bending loading and can be derived from moment limit solutions for the worst case of both in-plane (opening and closing) and out-of-plane moments. Calculations typically have a limited range of geometric applicability. In some instances it may be found that in order to meet the geometric limits a relatively large thickness is required (i.e. approaching or exceeding the nominal thickness) and hence alternative reference stress solutions should be considered, such as the use of the RSE-M approach [7].

At present there is no standard classical limit load solutions available for assessing plastic collapse of mitred bends and as such no FFP minimum required thicknesses for plastic collapse are included in the outage preparation activities. This is also the case for branches on bends and Y-pieces and if detailed assessment is required, FEA will be used. Hence, this is an area of development of standard FFP collapse solutions to expedite FFP required thickness calculations. However, not providing a FFP thickness is not a shortfall in the outage preparation activities because the thickness entered into the OMD will be based on that calculated using an appropriate design code methodology.

A reference stress solution for reducers is not readily available and hence they can simply be assessed as being two plain pipes. Two minimum required thicknesses should be determined - one for the small end and one for the large end. This neglects to account for any discontinuity stresses caused by the taper, but this is supported by Table A1 of PD 5500 [2] where wall bending stresses from pressure at the shell-tocone junction are classed as secondary and so would not contribute to plastic collapse.

\section{Ratcheting}

In order to prevent failure by ratcheting it is necessary to limit the calculated stress range so that it will shake down to elastic behaviour. Shakedown is achieved by setting the thickness so that the calculated stress range is equal to an allowable limit (dependent on the yield stress of the material used for the component). The methods of BS 806: 1993 [3] can be used to define the stress range and allowable limits, as they are well understood and are considered to give a reasonable and conservative estimate of the stress range. For non-creeping components the stress range should be limited to the sum of the yield stresses at room temperature and design temperature. A factor of 0.9 can be applied (unless the branch to main pipe diameter ratio was less than 0.3 in which case the 0.9 factor should not be applied). The calculations should be based on the normal operating pressure range and moment range as opposed to fault conditions. A combined (transverse, longitudinal and torsional) stress range for straight pipes and bends should be calculated. For bends, stress intensification factors should be applied to the components which comprise the combined stress. These can be obtained from [3], Figures 4.11.1 (2, 3, 4, and 5) for smooth bends and Figure 4.11.1(8) for mitred bends. Annex H of [3] can be used to calculate the combined stress range for branches. Where the limits of applicability for Annex $\mathrm{H}$ are not met, Section 4.11.5 of [3] can instead be used. The combined stress at the branch junction should consider the upstream and downstream moments in the main pipe and the moments in the branch. Stress intensification factors for branches can be obtained from Figure H.4.2 or Figure 4.11.1(6) of [3]. For all component types, if individual moment components are unknown, the equivalent moment should be 
considered to act entirely in-plane, out of plane and in torsion - the bounding case can then be used when determining the minimum required thickness.

No explicit method is available for assessing the stress range for Y-pieces, hence no minimum required thicknesses can be calculated for ratcheting. This is an area for potential further investigation. There is also no explicit method for reducers. Simplified methods can however be used, for example, reducers could be assessed as plain pipes but with a stress intensification factor (SIF) of 2 applied to the combined stress. A SIF of 2 has been chosen as it is the maximum SIF required for reducers in BS EN 13480-3 [1] and is likely to be bounding.

\section{Fatigue endurance}

Section 10.2 of BS EN 13480-3 [1] states that detailed fatigue analysis is not required if the number of alternating load cycles from all sources is less than 1,000. The number of load cycles from typical cold or hot shutdown to normal operating conditions expected during an AGR power station operating life is within these limits, numbering in the hundreds of cycles. Compared to the limit of 1,000 cycles, no detailed fatigue analysis would be required, assuming that the requirements of the rest of the code are met. It is noted, however, that not all power station systems may be governed solely by reactor cycles therefore to complement the above, further calculations may be performed in accordance with Section C.3 of PD 5500 [2]. The fatigue endurance can be evaluated at a stress range taken to be the allowable limit in the ratcheting calculations, as this will provide an upper bound if the thickness of the component is at least equal to the ratcheting minimum required thickness. Components identified as plain pipes, bends or reducers can be classified as 'Class D' details whist branches should be assessed as 'Class F' details, in accordance with PD 5500 [2]. The results obtained should demonstrate that for all components the fatigue life is large (in the many thousands of cycles). For branches on bends no specific consideration is given to fatigue endurance. This is due to the scantling thickness for branches on bends being determined by effectively multiplying stress intensification factors for the presence of the bend and the branch. It is judged that scantling thickness will be conservative. It is thus considered that compliance with the scantling thickness provided will lead to relatively low stress ranges within components and an adequate margin to failure by fatigue.

\section{DETERMINATION OF MATS}

A design code MAT is determined from the largest of the following minimum required thicknesses: i) pressure scantling, ii) sustained load and stress range flexibility, iii) buckling and iv) API fitness for service. A FFP MAT is determined from the largest of the following minimum required thicknesses: i) plastic collapse, ii) ratcheting, iii) buckling and iv) API fitness for service. A check for fatigue endurance should also be performed. This does not provide a minimum acceptable thickness, but the number of cycles that can be accommodated at a stress range equal to be the allowable limit in the ratcheting calculations. The MAT for inclusion in the OMD is usually determined as the larger of the design code and FFP minimum required thicknesses. This is not always the case, however, as there may be a reason to discount one over the other, for example, due to availability of data or known conservatisms leading to an overly pessimistic result.

\section{CASE STUDY - EXAMPLE MAT CALCULATION FOR A BEND}

\section{Geometry and operating conditions}

The example bend is a $90^{\circ}$ bend, located on $10^{\prime \prime}$ NB pipework which has a $103 / 4$ " $(273.1 \mathrm{~mm})$ outer diameter and a $0.281^{\prime \prime}(7.14 \mathrm{~mm})$ nominal thickness. The bend radius in this example was unknown, however, inspection of the design drawings suggests that it is a short radius welding elbow and therefore 
has a radius of $273.1 \mathrm{~mm}$. The (Fault) design temperature and pressure for the steam pipework were known and in excess of the (Normal) design temperature and pressure. Common with other MAT calculations fault pressures were assumed to be $110 \%$ of the Safety Relief Valve (SRV) lift pressure. It should be noted that the pipework being assessed is not subject to external pressure.

\section{System moments}

System moments were available from a pipe stress analysis model. The in-plane, out-of-plane, and torsional components have been combined to produce a single equivalent moment using a square root of the sum of the squares method. The sustained moment for design code flexibility calculations was extracted from the sustained (non intensified deadweight) load case. Moments from thermal range load cases have been used in stress range and ratcheting assessments. Fault moments for use in fitness for purpose, plastic collapse assessments were extracted from the bounding operation case. Bounding moments have been taken to be the largest moment in the run of pipework containing the component, bounded by stiffening features such as branches or valves. The system moments used in calculations for the bend were: Sustained $=\sim 10,000 \mathrm{Nm}$, Thermal Range $=\sim 10,500 \mathrm{Nm}$, Fault $=\sim 12,000 \mathrm{Nm}$.

\section{Material properties}

The following ambient and elevated temperature yield strengths and Ultimate Tensile Strengths have been used for Carbon Steel: $\sigma_{\mathrm{y} 20}=208 \mathrm{MPa}, \sigma_{\mathrm{y} 300}=145 \mathrm{MPa}, \sigma_{\mathrm{UTS} 20}=355 \mathrm{MPa}$ and $\sigma_{\mathrm{UTS} 300}=340 \mathrm{MPa}$.

\section{Design Code - Scantling}

The bends has been assessed in accordance with Section 6.2.3.1 of BS EN 13480-3 [1], with required thicknesses at the extrados and intrados determined using Equations 6.2.3-2 and 6.2.3-1 respectively. The larger of the two is the minimum required scantling thickness and is $4.9 \mathrm{~mm}$ in this case.

\section{Design Code - Flexibility}

Required thicknesses to meet the flexibility requirements of Section 12 of BS EN 13480-3 [1] were calculated for both sustained deadweight and internal pressure loading, and for stress ranges due to thermal expansion or alternating loads. Stress intensification factors were applied as defined in Table H.1 of the code. Equation 12.3.2-1 of BS EN 13480-3 [1] has been used to calculate the required thickness to avoid failure due to sustained loading, and takes account of axial pressure load and sustained mechanical loads (i.e. self weight). The design stress $f f$ was determined using Section 5.2 of the code.

Required thicknesses to avoid failure due to thermal expansion were calculated using equation 12.3.4-2 of BS EN 13480-3 [1], which limits the total axial stress due to sustained load and thermal expansion to the sum of the design stress at operating temperature and the allowable stress range, $f h+f a$. The allowable stress range $f a$ is defined in Equation 12.1.3-1 of the code and the allowable stress at maximum metal temperature $f h$ was determined using Equation 12.1.3-3. The stress range reduction factor $U$ was conservatively set to 1 such that the required thickness for stress range qualifies the pipework for 7,000 full pressure and temperature cycles. The minimum thickness to meet the sustained stress requirements of the design code was $7.0 \mathrm{~mm}$. The minimum thickness to meet the stress range requirements of the design code was $6.2 \mathrm{~mm}$.

\section{Fitness for Purpose - Plastic Collapse}

The minimum required thicknesses to resist plastic collapse for smooth bends was calculated. A limit solution for pressure only loading was used. A limiting moment for combined pressure and bending 
loading was also considered. This was derived from moment limit solutions for the worst case of both inplane (opening and closing) and out-of-plane moments. The calculations required the use of a bend factor, $\lambda$, which is $R t / r_{m}{ }^{2}$ where $R$ is the bend radius, $t$ is the pipe thickness, and $r_{m}$ is the pipe mean radius. The solution is only valid for bends with $\lambda$ between 0.1 and 1.5 and in this instance the example bend met this limit. The applied stress limit was two thirds of the lower bound flow stress.

In addition to the $\lambda$ limit, further geometric limits were imposed for each load combination. The minimum required thickness was determined by reducing the bend thickness until the ratio of the allowable moment to the actual moment becomes unity, or until the geometric limits of the code are met. For this bend the limiting factor was the $\mathrm{r}_{\mathrm{m}} / \mathrm{t}<20$ geometric limit for in-plane opening bending. At this limit the ratio of the allowable moment to the actual moment was 1.18 for the example bend. As these ratios are close to unity it was judged that using these minimum required thicknesses was valid. The minimum required thickness to resist plastic collapse was $6.7 \mathrm{~mm}$.

\section{Fitness for Purpose - Ratcheting}

The minimum required thickness to resist failure by ratcheting has been calculated using Section 4.11 of BS806: 1993 [3] and was based on the pressure range, design temperature and thermal system moments. The stress range was limited to 0.9 times the sum of $\sigma_{\mathrm{y} 20}$ and $\sigma_{\mathrm{y} 300}$. A combined (transverse, longitudinal and torsional) stress for was calculated. Stress intensification factors were applied to the stress components which comprise the combined. The minimum required thickness to resist failure by ratcheting was $6.6 \mathrm{~mm}$.

\section{Fatigue}

As the stress range remains elastic, fatigue endurance is considered to be long compared to the cyclic duty of the plant and hence failure by fatigue was not of concern. Insignificant fatigue has been confirmed by calculation to Section C.3 of PD 5500 [2]. Based on the allowable stress range and assuming a Class D weld, the fatigue endurance was in excess of 10,000 cycles. Given the number of expected start-up and shut down cycles and quadrant trips over the predicted life of the plant is in the order of 300-400, fatigue endurance is long.

\section{Determination of the MAT}

The minimum required thicknesses determined for the bend are as follows:
Design code scantling
$4.9 \mathrm{~mm}$
FFP plastic collapse
$6.7 \mathrm{~mm}$
Design code sustained stress $\quad 7.0 \mathrm{~mm}$
FFP stress range
$6.6 \mathrm{~mm}$
Design code stress range
$6.2 \mathrm{~mm}$
External pressure
N/A

The design code required thickness is the largest of the three minimum required thicknesses on the left and hence is $7.0 \mathrm{~mm}$. The FFP minimum required thickness is the largest of the two values on the right and hence is $6.7 \mathrm{~mm}$. At these thicknesses, fatigue has been demonstrated to not be of concern. Given the magnitude of these thicknesses, incorporation of the API 579 required thickness was considered to not be of benefit. The MAT for entry into the OMD was taken as the largest of the deign code and FFP minimum required thicknesses and hence was $7.0 \mathrm{~mm}$.

\section{CONCLUSIONS}

The purpose and objective of this paper is to provide an overview of the statutory outage process applicable to nuclear power plants in the UK, with a particular focus on the structural integrity 
assessments required prior to, during and after the statutory outage. The provision and use of Minimum Acceptable Thickness for defect free components has been used as an example of such assessments. These calculations involve the use of design code and FFP methodologies which are applied in a nontraditional sense, whereby thicknesses are determined as opposed to stresses or stress ranges. The key conclusions which can be drawn are as follows:

- Statutory outages are an important part of ensuring the continued safe operation of UK nuclear power plants and effective planning prior to commencement of these outages is essential;

- Nuclear safety is of utmost importance and structural integrity assessments are a key part in demonstrating the continued safe operation of the plant both prior to, during and following statutory outages;

- One aspect of these structural integrity assessments involves calculating Minimum Acceptable Thickness for comparison with values measured during the outage. For items which are considered to be under thickness, further assessments are required to consider their continued operation as is or repair/replacement prior to their return to service;

- It is important there is a consistent approach across the AGR fleet when determining Minimum Acceptable Thicknesses. This is typically achieved via design code and Fitness for Purpose based assessments;

- Whereas such assessment would typically be performed in order to compare stresses or stress ranges with stress and stress range allowables, the equations often require rearrangement or an iterative process followed so a thickness can be determined when the stress or stress range is equal to the code allowable;

- Whilst the calculation route is well understood for a majority of component types, further work is required to develop standard FFP collapse solutions of mitred bends and for the FFP collapse and FFP stress range assessments of branches on bends and Y-pieces. The current alternative is to use FEA which could usefully be replaced by standard solutions to increase the efficiency of outage preparation activities.

\section{ACKNOWLEDGMENTS}

We would like to thank EDF Energy for input into discussions that contributed to this paper.

\section{REFERENCES}

[1] British Standards Institution, BS EN 13480-3:2012, Metallic industrial piping - Part 3: Design and calculation, August 2012.

[2] British Standards Institution, PD $5500+$ A2:2013, Specification for unfired fusion welded pressure vessels, November 2013.

[3] British Standards Institution, BS 806: 1993, Specification for design and construction of ferrous piping installations for and in connection with land boilers, September 1993.

[4] British Standards Institution, BS 1640-3: 1968 Incorporating amendment issued March 1972, Specification for Steel butt-welding pipe fittings - For the petroleum industry - Part 3: Wrought carbon and ferritic alloy steel fittings - Metric units, March 1972.

[5] BSI Standards Publication, BS EN 13445-3:2014, Unfired Pressure Vessels - Part 3: Design, September 2014.

[6] The American Society of Mechanical Engineers, API 579-1/ASME FFS-1, Fitness for Service, Second Edition, June 2007.

[7] S. Marie et al, French RSE-M and RCC-MR Code Appendices for Flaw Analysis: Presentation of the Fracture Parameters Calculation - Part IV: Cracked Elbows, International Journal of Pressure Vessels and Piping 84 (2007) 659-686. 